\title{
Possibility, impossibility, and cheat sensitivity of quantum-bit string commitment
}

\author{
Harry Buhrman, ${ }^{1}$ Matthias Christandl, ${ }^{2,3, *}$ Patrick Hayden, ${ }^{4}$ Hoi-Kwong Lo, ${ }^{5}$ and Stephanie Wehner ${ }^{1,6, \dagger}$ \\ ${ }^{1}$ CWI, Kruislaan 413, 1098 SJ Amsterdam, The Netherlands \\ ${ }^{2}$ DAMTP, University of Cambridge, Wilberforce Road, Cambridge, CB3 OWA, United Kingdom \\ ${ }^{3}$ Arnold Sommerfeld Center for Theoretical Physics, Faculty of Physics, Ludwig-Maximilians-University Munich, Theresienstrasse 37, \\ 80333 Munich, Germany \\ ${ }^{4}$ School of Computer Science, McGill University, Montreal, Canada \\ ${ }^{5}$ Department of ECE and Physics, University of Toronto, Canada M5G $3 G 4$ \\ ${ }^{6}$ California Institute of Technology, 1200 East California Boulevard, Pasadena California 91125, USA
}

(Received 8 November 2007; published 11 August 2008)

\begin{abstract}
Unconditionally secure nonrelativistic bit commitment is known to be impossible in both the classical and the quantum worlds. But when committing to a string of $n$ bits at once, how far can we stretch the quantum limits? In this paper, we introduce a framework for quantum schemes where Alice commits a string of $n$ bits to Bob in such a way that she can only cheat on $a$ bits and Bob can learn at most $b$ bits of information before the reveal phase. Our results are twofold: we show by an explicit construction that in the traditional approach, where the reveal and guess probabilities form the security criteria, no good schemes can exist: $a+b$ is at least $n$. If, however, we use a more liberal criterion of security, the accessible information, we construct schemes where $a=4 \log _{2} n+O(1)$ and $b=4$, which is impossible classically. We furthermore present a cheat-sensitive quantum bit string commitment protocol for which we give an explicit tradeoff between Bob's ability to gain information about the committed string, and the probability of him being detected cheating.
\end{abstract}

DOI: 10.1103/PhysRevA.78.022316

PACS number(s): 03.67.Dd

\section{INTRODUCTION}

Commitments play an important role in modern day cryptography. Informally, a commitment allows one party to prove that she has made up her mind and cannot change it, while hiding the actual decision until later. Imagine two mutually distrustful parties Alice and Bob at distant locations. They can only communicate over a channel, but want to play the following game: Alice secretly chooses a bit $x$. Bob wants to be sure that Alice indeed has made her choice. At the same time, Alice wants to keep $x$ hidden from Bob until she decides to reveal $x$. To convince Bob that she made up her mind, Alice sends Bob a commitment. From the commitment alone, Bob cannot deduce $x$. At a later time, Alice reveals $x$ and enables Bob to open the commitment. Bob can now check if Alice is telling the truth. This scenario is known as bit commitment.

Bit commitment is a very powerful cryptographic primitive with a wide range of applications. It has been shown that quantum oblivious transfer (QOT) [1] can be achieved provided there exists a secure bit commitment scheme [2,3]. In turn, oblivious transfer is known to be sufficient for solving the general problem of secure two-party computation $[4,5]$. Commitments are also useful for constructing zeroknowledge proofs [6]. Furthermore, a bit commitment protocol can be used to implement secure coin tossing [7]. Classically, unconditionally secure bit commitment is known to be impossible. Unfortunately after several quantum schemes were suggested [8-10], nonrelativistic quantum bit commitment has also been shown to be impossible [11-16]. Only

\footnotetext{
*matthias.christandl@qubit.org

†wehner@caltech.edu
}

very limited degrees of concealment and binding can be achieved [17]. In the face of these negative statements, what can we still hope to achieve?

\section{A. String commitment}

Here we take a different approach and look at the task of committing to a string of $n$ bits at once in the setting where Alice and Bob have unbounded resources. Since perfect bit commitment is impossible, perfect string commitment is impossible, too. However, is it possible to design meaningful string commitment schemes when we allow for a small ability to cheat on both Alice's and Bob's side? To make this question precise, we introduce a framework for the classification of string commitments in terms of the length $n$ of the string, Alice's ability to cheat on $a$ bits and Bob's ability to acquire $b$ bits of information before the reveal phase. Instead of asking for a perfectly binding commitment, we allow Alice to reveal up to $2^{a}$ strings successfully: Bob will accept any such string as a valid opening of the commitment. Formally, we demand that $\Sigma_{x \in\{0,1\}} p_{x}^{A} \leq 2^{a}$, where $p_{x}^{A}$ is the probability that Alice successfully reveals string $x$ during the reveal phase. Contrary to classical computing, Alice can always choose to perform a superposition of string commitments without Bob's knowledge. Thus even for a perfectly binding string commitment we would only demand $\Sigma_{x \in\{0,1\}} p_{x}^{A} \leq 1$, since a strategy based on superpositions is indistinguishable from the "classical" honest behavior of choosing a string beforehand and then committing to it. At the same time, we relax Bob's security condition, and allow him to acquire at most $b$ bits of information before the reveal phase. The nature of his security definition is crucial to our investigation: If $b$ determines a bound on his probability to guess Alice's string, then we prove that $a+b$ is at least $n$ (up 
to a small constant). We write $(n, a, b)$-QBSC for a quantum bit string commitment protocol where the string has length $n$ and $a$ and $b$ are the security parameters for Alice and Bob as explained in detail below. In Sec. II, we show:

Impossibility of $(n, a, b)$ QBSC. Every $(n, a, b)$-QBSC scheme with $a+b+c<n$ is insecure, where $c \approx 7.61$.

Our proof makes use of privacy amplification with twouniversal hash functions. If the protocol is executed multiple times in parallel, we prove that any quantum bit string commitment protocol with $a+b<n$ is insecure. We refer to these results as "impossibilities," as they show that QBSCs offer almost no advantage over the trivial classical protocol: Alice first sends $b$ bits of the $n$ bit string to Bob during the commit phase, and then supplies him with the remaining $n-b$ bits in the reveal phase.

The second part of the paper is devoted to the "possibility" of QBSC. If we weaken our standard of security and measure Bob's information gain in terms of the accessible information, it becomes possible to construct meaningful QBSC protocols with $a=4 \log _{2} n+O(1)$ and $b=4$. Our protocols are based on the effect of locking classical information in quantum states [18]. This surprising effect shows that given an initial shared quantum state, the transmission of $l$ classical bits can increase the total amount of correlation by more than $l$ bits. In Sec. III, we show the following.

Possibility of $(n, a, b)-\mathrm{QBSC}_{I_{\mathrm{acc}}}$. For $n \geq 3$, there exist $\left[n, 4 \log _{2} n+O(1), 4\right]-\mathrm{QBSC}_{I_{\text {acc }}}$ protocols.

We then turn our attention to a specific $(n, 1, n / 2)$ $-\mathrm{QBSC}_{I_{\text {acc }}}$ protocol. Note that Bob is able to gain quite large amounts of mutual information $\left(\frac{n}{2}\right.$ bits $)$ with Alice's committed string before the reveal phase. As we show in this paper, however, Bob's cheating will be detected by Alice with positive probability if he performs any measurement that leads to a positive information gain; in other words, the protocol is cheat-sensitive against Bob. More precisely, we give an explicit tradeoff between Bob's information gain and Alice's ability to catch him cheating. In Sec. IV, we show the following.

Cheat-sensitive $(n, 1, n / 2)-\mathrm{QBSC}_{I_{\mathrm{acc}}}$. There exists an $(n, 1, n / 2)-\mathrm{QBSC}_{I_{\text {acc }}}$ that is cheat-sensitive against Bob: if Bob is detected cheating with probability less than $p$, then the mutual information that he gained by measurement before the reveal phase is bounded by $4 \sqrt{p} \log _{2} d+2 \mu(2 \sqrt{p})$ where $\mu(x)=\min \left\{-x \log _{2} x, 1 / e\right\}$.

\section{B. Related work}

To obtain bit commitment, different restrictions have been introduced into the model. Salvail [19] showed that, for any fixed $n$, secure bit commitment is possible provided that the sender is not able to perform generalized measurements on more then $n$ qubits coherently. Large $n$ coherent measurements are not yet feasible, so his result provides an implementation which is secure under a plausible technological assumption. DiVincenzo, Smolin, and Terhal took a different approach [20], showing that if the bit commitment is forced to be ancilla-free, a type of asymptotic security is still possible. Bit commitment is also possible if the adversary's quantum storage is bounded [21-23] or noisy [24]. Classi- cally, introducing restrictions can also open new possibilities. Cachin, Crépeau, and Marcil have shown how to implement bit commitment via oblivious transfer under the assumption that the size of the receiver's memory is bounded [25]. Furthermore, the assumption of a noisy channel can be sufficient for oblivious transfer [26,27]. A cryptographic task-called cheat-sensitive bit commitment-has been studied by Hardy and Kent [28], as well as Aharanov, Ta-Shma, Vazirani, and Yao [29]: no restrictions are placed on the adversary initially, but an honest party should stand a good chance of catching a cheater. Kent also showed that bit commitment can be achieved using relativistic constraints [30].

Classically, string commitment is directly linked to bit commitment and no interesting protocols are possible. Kent [31] first asked what kind of quantum string commitment (QBSC) can be achieved. He gave a protocol under the restrictive assumption that Alice does not commit to a superposition [32]. His protocol was modified for experimental purposes by Tsurumaru [33].

\section{PRELIMINARIES}

\section{A. Framework}

We first formalize the notion of quantum string commitments in a quantum setting.

Definition 1. An $(n, a, b)$-quantum bit string commitment (QBSC) is a quantum communication protocol between two parties, Alice (the committer) and Bob (the receiver), which consists of two phases and two security requirements.

Commit phase. Assume that both parties are honest. Alice chooses a string $x \in\{0,1\}^{n}$ with probability $p_{x}$. Alice and Bob communicate and at the end Bob holds state $\rho_{x}$.

Reveal phase. If both parties are honest, Alice and Bob communicate and at the end Bob learns $x$. Bob accepts.

Concealing. If Alice is honest, $\Sigma_{x \in\{0,1\}^{n}} p_{x \mid x}^{B} \leq 2^{b}$, where $p_{x \mid x}^{B}$ is the probability that Bob correctly guesses $x$ before the reveal phase.

Binding. If Bob is honest, then for all commitments of Alice: $\Sigma_{x \in\{0,1\}^{n}} p_{x}^{A} \leq 2^{a}$, where $p_{x}^{A}$ is the probability that Alice successfully reveals $x$.

We say that Alice successfully reveals a string $x$ if Bob accepts the opening of $x$, i.e., he performs a test depending on the individual protocol to check Alice's honesty and concludes that she was indeed honest. Note that quantumly, Alice can always commit to a superposition of different strings without being detected. Thus even for a perfectly binding bit string commitment (i.e., $a=0$ ) we only demand that $\Sigma_{x \in\{0,1\}^{n}} p_{x}^{A} \leq 1$, whereas classically one wants that $p_{x^{\prime}}^{A}$ $=\delta_{x, x^{\prime}}$. Note that our concealing definition reflects Bob's $a$ priori knowledge about $x$. We choose an a priori uniform distribution (i.e., $\left.p_{x}=2^{-n}\right)$ for $(n, a, b)$-QBSCs, which naturally comes from the fact that we consider $n$-bit strings. A generalization to any $\left(P_{X}, a, b\right)$-QBSC where $P_{X}$ is an arbitrary distribution is possible but omitted in order not to obscure our main line of argument. Instead of Bob's guessing probability, one can take any information measure $B$ to express the security against Bob. In general, we consider an $(n, a, b)-\mathrm{QBSC}_{B}$ where the new concealing condition $B(\mathcal{E})$ 
$\leq b$ holds for any ensemble $\mathcal{E}=\left\{p_{x}, \rho_{x}\right\}$ that Bob can obtain by a cheating strategy. In the latter part of this paper we show that for $B$ being the accessible information nontrivial protocols, i.e., protocols with $a+b \ll n$, exist. The accessible information is defined as $I_{\text {acc }}(\mathcal{E})=\max _{M} I(X ; Y)$, where $P_{X}$ is the prior distribution of the random variable $X, Y$ is the random variable of the outcome of Bob's measurement on $\mathcal{E}$, and the maximization is taken over all measurements $M$.

\section{B. Model}

We work in the model of two-party nonrelativistic quantum protocols of Yao [2] and then simplified by Lo and Chau [12] which is usually adopted in this context. Here, any twoparty quantum protocol can be regarded as a pair of quantum machines (Alice and Bob), interacting through a quantum channel. Consider the product of three Hilbert spaces $\mathcal{H}_{A}$, $\mathcal{H}_{B}$, and $\mathcal{H}_{C}$ of bounded dimensions representing the Hilbert spaces of Alice's and Bob's machines and the channel, respectively. Without loss of generality, we assume that each machine is initially in a specified pure state. Alice and Bob perform a number of rounds of communication over the channel. Each such round can be modeled as a unitary transformation on $\mathcal{H}_{A} \otimes \mathcal{H}_{C}$ and $\mathcal{H}_{B} \otimes \mathcal{H}_{C}$, respectively. Since the protocol is known to both Alice and Bob, they know the set of possible unitary transformations used in the protocol. We assume that Alice and Bob are in possession of both a quantum computer and a quantum storage device. This enables them to add ancillae to the quantum machine and use reversible unitary operations to replace measurements. By doing so, Alice and Bob can delay measurements and thus we can limit ourselves to protocols where both parties only measure at the very end. Moreover, any classical computation or communication that may occur can be simulated by a quantum computer. Furthermore, any probabilistic operation can be modeled as an operation that is conditional on the outcome of a coin flip. Instead of a classical coin, we can use a quantum coin and in this way keep the whole system fully quantum mechanical.

\section{Tools}

We now gather the essential ingredients for our proof. First, we show that every $(n, a, b)$-QBSC is an $(n, a, b)-\mathrm{QBSC}_{\xi}$. The security measure $\xi(\mathcal{E})$ is defined by

$$
\xi(\mathcal{E}) \equiv n-H_{2}\left(\rho_{A B} \mid \rho\right),
$$

where $\rho_{A B}=\Sigma_{x} p_{x}|x\rangle\langle x| \otimes \rho_{x}$ and $\rho=\Sigma_{x} p_{x} \rho_{x}$ only depend on the ensemble $\mathcal{E}=\left\{p_{x}, \rho_{x}\right\}$. $H_{2}$ is an entropic quantity defined in Ref. [34] $H_{2}\left(\rho^{A B} \mid \rho\right) \equiv-\log _{2} \operatorname{Tr}\left[\left(\mathbb{1} \otimes \rho^{-1 / 2}\right) \rho_{A B}\right]^{2}$. This quantity is directly connected to Bob's maximal average probability of successfully guessing the string.

Lemma 1. Bob's maximal average probability of successfully guessing the committed string, i.e., $\sup _{M} \Sigma_{x} p_{x} p_{x \mid x}^{B, M}$ where $M$ ranges over all measurements and $p_{y \mid x}^{B, M}$ is the conditional probability of guessing $y$ given $\rho_{x}$, obeys

$$
\sup _{M} \sum_{x} p_{x} p_{x \mid x}^{B, M} \geq 2^{-H_{2}\left(\rho_{A B} \mid \rho\right)} .
$$

Proof. By definition the maximum average guessing probability is lower bounded by the average guessing probability for a particular measurement strategy. We choose the squareroot measurement which has operators $M_{x}=p_{x} \rho^{-1 / 2} \rho_{x} \rho^{-1 / 2}$. $p_{x \mid x}^{B}=\operatorname{Tr}\left(M_{x} \rho_{x}\right)$ is the probability that Bob guesses $x$ given $\rho_{x}$, hence

$$
\begin{aligned}
\log _{2} \sum_{x} p_{x} p_{x \mid x}^{B, \max } & \geq \log _{2} \sum_{x} p_{x}^{2} \operatorname{Tr}\left(\rho^{-1 / 2} \rho_{x} \rho^{-1 / 2} \rho_{x}\right) \\
& =\log _{2} \operatorname{Tr}\left\{\left[\left(\mathbb{I} \otimes \rho^{-1 / 2}\right) \rho_{A B}\right]^{2}\right\}=-H_{2}\left(\rho_{A B} \mid \rho\right)
\end{aligned}
$$

Related estimates were derived in Ref. [35]. For the uniform distribution $p_{x}=2^{-n}$ we have from the concealing condition that $\sum_{x} p_{x \mid x}^{B} \leq 2^{b}$ which by lemma 1 implies $\xi(\mathcal{E}) \leq b$ and hence the following lemma.

Lemma 2. Every $(n, a, b)-\mathrm{QBSC}$ is an $(n, a, b)-Q B S C_{\xi}$.

Furthermore, we make use of the following theorem, known as privacy amplification against a quantum adversary. In our case, Bob holds the quantum memory and privacy amplification is used to find Alice's attack.

Theorem 1 [Th. 5.5.1 in Ref. [34] (see also Ref. [36])]. Let $\mathcal{G}$ be a class of two-universal hash functions from $\{0,1\}^{n}$ to $\{0,1\}^{s}$. Application of $g \in \mathcal{G}$ to the random variable $X$ maps the ensemble $\mathcal{E}=\left\{p_{x}, \rho_{x}\right\}$ to $\mathcal{E}_{g}=\left\{q_{y}^{g}, \sigma_{y}^{g}\right\}$ with probabilities $q_{y}^{g}=\Sigma_{x \in g^{-1}(y)} p_{x}$ and quantum states $\sigma_{y}^{g}=\sum_{x \in g^{-1}(y)} p_{x} \rho_{x}$. Then

$$
\frac{1}{|\mathcal{G}|} \sum_{g \in \mathcal{G}} d\left(\mathcal{E}_{g}\right) \leq \frac{1}{2} 2^{-1 / 2\left[H_{2}\left(\rho_{A B} \mid \rho\right)-s\right]},
$$

where $d(\mathcal{E}) \equiv \delta\left(\sum_{x} p_{x}|x\rangle\langle x| \otimes \rho_{x}, \mathbb{I} / 2^{n} \otimes \rho\right)$ [and similarly for $\left.d\left(\mathcal{E}_{g}\right)\right]$ and $\delta(\alpha, \beta) \equiv \frac{1}{2}\|\alpha-\beta\|_{1}$ with $\|A\|_{1}=\operatorname{Tr} \sqrt{A^{\dagger} A}$.

Finally, the following reasoning, previously used to prove the impossibility of quantum bit commitment [11,12], will be essential: Suppose $\rho_{0}$ and $\rho_{1}$ are density operators that correspond to a commitment of a " 0 " or a " 1 ," respectively. Let $\left|\phi_{0}\right\rangle$ and $\left|\phi_{1}\right\rangle$ be the corresponding purifications on the joint system of Alice and Bob. If $\rho_{0}$ equals $\rho_{1}$ then Alice can find a local unitary transformation $U$ that she can apply to her part of the system and satisfying $\left|\phi_{1}\right\rangle=U \otimes \mathbb{I}\left|\phi_{0}\right\rangle$. This enables Alice to change the total state from $\left|\phi_{0}\right\rangle$ to $\left|\phi_{1}\right\rangle$ and thus cheat. This also holds in an approximate sense [11], used here in the following form.

Lemma 3. Let $\delta\left(\rho_{0}, \rho_{1}\right) \leq \epsilon$ and assume that the bitcommitment protocol is error-free if both parties are honest. Then there is a method for Alice to cheat such that the probability of successfully revealing a 0 given that she committed to a 1 is greater or equal to $1-\sqrt{2 \epsilon}$.

Proof. $\delta\left(\rho_{0}, \rho_{1}\right) \leq \epsilon$ implies $F\left(\rho_{0}, \rho_{1}\right) \geq 1-\epsilon . F$ is the fidelity of two quantum states, which equals $\max _{U} \mid\left\langle\phi_{0}\right| U$ $\otimes \mathbb{I}\left|\phi_{1}\right\rangle \mid$ by Uhlmann's theorem. Here, $\left|\phi_{0}\right\rangle$ and $\left|\phi_{1}\right\rangle$ are the joint states after the commit phase and the maximization ranges over all unitaries $U$ on Alice's (i.e., the purification) side. Let $\left|\psi_{0}\right\rangle=U \otimes \mathbb{I}\left|\phi_{1}\right\rangle$ for a $U$ achieving the maximization. Then

$\delta\left(\left|\phi_{0}\right\rangle\left\langle\phi_{0}|,| \psi_{0}\right\rangle\left\langle\psi_{0}\right|\right)=\sqrt{1-\left|\left\langle\phi_{0} \mid \psi_{0}\right\rangle\right|^{2}} \leq \sqrt{1-(1-\epsilon)^{2}} \leq \sqrt{2 \epsilon}$.

If both parties are honest, the reveal phase can be regarded as a measurement resulting in a distribution $P_{Y}\left(P_{Z}\right)$ if $\left|\phi_{0}\right\rangle$ $\left(\left|\psi_{0}\right\rangle\right)$ was the state before the reveal phase. The random 


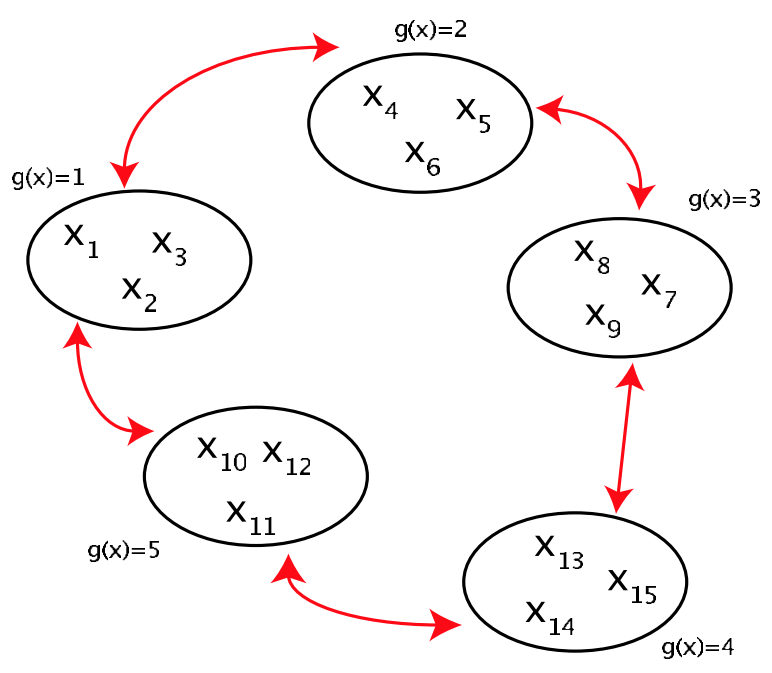

FIG. 1. (Color online) Moving from $y$ to $y^{\prime}$.

variables $Y$ and $Z$ carry the opened bit or the value "reject $(r)$." Since the trace distance does not increase under measurements $\delta\left(P_{Y}, P_{Z}\right) \leq \delta\left(\left|\phi_{0}\right\rangle\left\langle\phi_{0}|,| \psi_{0}\right\rangle\left\langle\psi_{0}\right|\right) \leq \sqrt{2 \epsilon}$. Hence $\frac{1}{2}\left[\left|P_{Y}(0)-P_{Z}(0)\right|+\left|P_{Y}(1)-P_{Z}(1)\right|+\left|P_{Y}(r)-P_{Z}(r)\right|\right] \leq \sqrt{2 \epsilon}$.

Since $\left|\phi_{0}\right\rangle$ corresponds to Alice's honest commitment to 0 we have $P_{Y}(0)=1, P_{Y}(1)=P_{Y}(r)=0$, and hence $P_{Z}(0) \geq 1$ $-\sqrt{2 \epsilon}$.

\section{IMPOSSIBILITY}

The proof of our impossibility result consists of three steps: in the previous section, we saw that any $(n, a, b)$-QBSC is also an $(n, a, b)-\mathrm{QBSC}_{\xi}$ with the security measure $\xi(\mathcal{E})$ defined by Eq. (1). Below, we prove that an $(n, a, b)-\mathrm{QBSC}_{\xi}$ can only exist for values $a, b$, and $n$ obeying $a+b+c \geq n$, where $c$ is a small constant independent of $a, b$, and $n$. This in turn implies the impossibility of an $(n, a, b)$-QBSC for such parameters. At the end of this section we show that many executions of the protocol can only be secure if $a+b \geq n$.

The intuition behind our main argument is simple. To cheat, Alice first chooses a two-universal hash function $g$. She then commits to a superposition of all strings for which $g(x)=y$ for a specific $y$. We know from the privacy amplification theorem above, however, that even though Bob may gain some knowledge about $x$, he is entirely ignorant about $y$. But then Alice can change her mind and move to a different set of strings for which $g(x)=y^{\prime}$ with $y \neq y^{\prime}$ as we saw above. Figure 1 illustrates this idea.

Theorem 2. $(n, a, b)-\mathrm{QBSC}_{\xi}$ schemes, and thus also $(n, a, b)$-QBSC schemes, with $a+b+c<n$ do not exist. $c$ is a constant equal to $5 \log _{2} 5-4 \approx 7.61$.

Proof. Consider an $(n, a, b)-\mathrm{QBSC}_{\xi}$ and the case where both Alice and Bob are honest. Alice committed to $x$. We denote the joint state of the Alice-Bob-channel system $\mathcal{H}_{A}$ $\otimes \mathcal{H}_{B} \otimes \mathcal{H}_{C}$ after the commit phase by $\left|\phi_{x}\right\rangle$ for input state $|x\rangle$. Let $\rho_{x}$ be Bob's reduced density matrix and let $\mathcal{E}=\left\{p_{x}, \rho_{x}\right\}$, where $p_{x}=2^{-n}$.

Assuming that Bob is honest, we will give a cheating strategy for Alice in the case where $a+b+5 \log _{2} 5-4<n$.
The strategy will depend on the two-universal hash function $g: \mathcal{X}=\{0,1\}^{n} \rightarrow \mathcal{Y}=\{0,1\}^{n-m}$, for appropriately chosen $m$. Alice picks a $y \in \mathcal{Y}$ and prepares the state $\left(\sum_{x \in g^{-1}(y)}|x\rangle|x\rangle\right) / \sqrt{\left|g^{-1}(y)\right|}$. She then gives the second half of this state as input to the protocol and stays honest for the rest of the commit phase. The joint state of Alice and Bob at the end of the commit phase is thus $\left|\psi_{y}^{g}\right\rangle$ $=\left(\Sigma_{x \in g^{-1}(y)}|x\rangle\left|\phi_{x}\right\rangle\right) / \sqrt{\left|g^{-1}(y)\right|}$. The reduced states on Bob's side are $\sigma_{y}^{g}=\frac{1}{q_{y}^{g}} \sum_{x \in g^{-1}(y)} p_{x} \rho_{x}$ with probability $q_{y}^{g}=\Sigma_{x \in g^{-1}(y)} p_{x}$. We denote this ensemble by $\mathcal{E}_{g}$. Let $\sigma=\sigma^{g}=\Sigma_{y} q_{y}^{g} \sigma_{y}^{g}$ for all $g$.

We now apply theorem 1 with $s=n-m$ and $\xi(\mathcal{E}) \leq b$ to obtain $\frac{1}{|\mathcal{G}|} \Sigma_{g \in \mathcal{G}} d\left(\mathcal{E}_{g}\right) \leq \epsilon$, where $\epsilon=\frac{1}{2} 2^{-1 / 2(m-b)}$. Hence, there is at least one $g$ such that $d\left(\mathcal{E}_{g}\right) \leq \epsilon$; intuitively, this means that Bob knows only very little about the value of $g(x)$. This $g$ defines Alice's cheating strategy. It is straightforward to verify that $d\left(\mathcal{E}_{g}\right) \leq \epsilon$ implies

$$
2^{-(n-m)} \sum_{y} \delta\left(\sigma, \sigma_{y}^{g}\right) \leq 2 \epsilon
$$

Let us therefore assume without loss of generality that Alice chooses $y_{0} \in \mathcal{Y}$ with $\delta\left(\sigma, \sigma_{y_{0}}^{g}\right) \leq 2 \epsilon$.

Clearly, the probability to successfully reveal some $x$ in $g^{-1}(y)$ given $\left|\psi_{y}^{g}\right\rangle$ is one. Note that Alice learns $x$, but cannot pick it: she committed to a superposition and $x$ is chosen randomly by measurement. Thus the probability to reveal $y$ [i.e., to reveal an $x$ such that $y=g(x)]$ given $\left|\psi_{y}^{g}\right\rangle$ successfully is 1 . Let $\widetilde{p}_{x}$ and $\widetilde{q}_{y}^{g}$ denote the probabilities to successfully reveal $x$ and $y$, respectively, and $\tilde{p}_{x \mid y}^{g}$ be the conditional probability to successfully reveal $x$, given $y$. We have

$$
\sum_{x} \tilde{p}_{x}=\sum_{y} \widetilde{q}_{y}^{g} \sum_{x \in g^{-1}(y)} \widetilde{p}_{x \mid y}^{g} \geq \sum_{y} \widetilde{q}_{y}^{g} .
$$

Recall that Alice can transform $\left|\psi_{y_{0}}^{g}\right\rangle$ approximately into $\left|\psi_{y}^{g}\right\rangle$ if $\sigma_{y_{0}}^{g}$ is sufficiently close to $\sigma_{y}^{g}$ by applying local transformations to her part alone. It follows from lemma 3 that we can estimate the probability of revealing $y$, given that the state was really $\left|\psi_{y_{0}}\right\rangle$. Since this reasoning applies to all $y$, on average, we have

$$
\begin{aligned}
\sum_{y} \widetilde{q}_{y}^{g} \geq & \sum_{y}\left[1-2^{1 / 2} \delta\left(\sigma_{y_{0}}^{g}, \sigma_{y}^{g}\right)^{1 / 2}\right] \geq 2^{n-m} \\
& -2^{1 / 2} 2^{n-m}\left[2^{m-n} \sum_{y} \delta\left(\sigma_{y_{0}}^{g}, \sigma_{y}^{g}\right)\right]^{1 / 2} \\
\geq & 2^{n-m}\left(1-2^{1 / 2}\left\{2^{m-n}\left[\sum_{y} \delta\left(\sigma_{y_{0}}^{g}, \sigma\right)+\delta\left(\sigma, \sigma_{y}^{g}\right)\right]\right\}^{1 / 2}\right) \\
\geq & 2^{n-m}\left[1-2(2 \varepsilon)^{1 / 2}\right],
\end{aligned}
$$

where the first inequality follows from lemma 3, the second from Jensen's inequality and the concavity of the square root function, the third from the triangle inequality and the fourth from Eq. (3) and $\delta\left(\sigma_{y_{0}}^{g}, \sigma\right) \leq 2 \varepsilon$. Recall that to be secure against Alice, we require $2^{a} \geq 2^{n-m}\left[1-2(2 \varepsilon)^{1 / 2}\right]$. We insert $\epsilon=\frac{1}{2} 2^{-1 / 2(m-b)}$, define $m=b+\gamma$ and take the logarithm on both sides to get 


$$
a+b+\delta \geq n,
$$

where $\delta=\gamma-\log _{2}\left(1-2^{-\gamma / 4+1}\right)$. Keeping in mind that $1-2^{-\gamma / 4+1}>0$ (or, equivalently, $\gamma>4$ ), we find that the minimum value of $\delta$ for which Eq. (4) is satisfied is $\delta$ $=5 \log _{2} 5-4$ and arises from $\gamma=4\left(\log _{2} 5-1\right)$. Thus, no $(n, a, b)-\mathrm{QBSC}_{\xi}$ with $a+b+5 \log _{2} 5-4<n$ exists.

Since the constant $c$ does not depend on $a, b$, and $n$, multiple parallel executions of the protocol in the form of multiple simultaneous commit phases followed by the corresponding opening phases, can only be secure if $a+b \geq n$ :

Proposition 1. Let $P$ be an $(n, a, b)-\mathrm{QBSC}_{\xi}$ or $(n, a, b)$-QBSC. The $m$-fold parallel execution of $P$ will be insecure if $a+b<n-c / m$. In particular, no $(n, a, b)-\mathrm{QBSC}_{\xi}$ or $(n, a, b)$-QBSC with $a+b<n$ can be executed securely an arbitrary number of times in parallel. Furthermore, no $(n, a, b)-\mathrm{QBSC}_{\chi}$ with $a+b<n$ and $\chi$ the Holevo information can be executed securely an arbitrary number of times in parallel.

Proof. In the following, we assume without loss of generality that $a$ and $b$ are the smallest cheat parameters for $P$. Let $Q$ denote the $\left(n m, a_{m}, b_{m}\right)$-QBSC or $_{\xi}\left(n m, a_{m}, b_{m}\right)$-QBSC protocol obtained by executing $P m$ times in parallel. By theorem 2, $Q$ is insecure if $a_{m}+b_{m}<n m-c$. Since $a$ and $b$ were assumed to be the smallest cheat parameters for $P$, the product cheating attack by Alice and Bob lead to the estimates $a_{m} \geq a m$ and $b_{m} \geq b m$, respectively. Therefore, the $m$-fold execution of $P$ is insecure, if $a m+b m \leq a_{m}+b_{m}$ $<n m-c$ or $a+b<n-c / m$.

In order to prove the result about Holevo information QBSC, we will use a slightly different characterization of privacy amplification in the proof of theorem 2 . In this characterization, the right-hand side of Eq. (2) is replaced by $\kappa$ $+2^{-1 / 2\left[H_{\min }^{\kappa}\left(\rho_{A B} \mid \rho_{B}\right)-s\right]}$ for an arbitrary $\kappa>0$ (Ref. [34] Corollary 5.6.1). Going through the proof with this change in mind, one sees that $Q$ is not a $\left(n m, a_{m}, b_{m}\right)-\mathrm{QBSC} \Xi$ for $\Xi(\widetilde{\mathcal{E}})=n m-H_{\min }^{\kappa}\left(\tilde{\rho}_{A B} \mid \widetilde{\rho}\right)$ if $a_{m}+b_{m}+\delta \leq m n$. Here, $\widetilde{\mathcal{E}}$ is the ensemble corresponding to $Q$ and $\tilde{\rho}_{A B}$ and $\tilde{\rho}$ the related states; $\delta \equiv \delta(\kappa)$ is a positive constant independent of $n$. Since $\widetilde{\mathcal{E}}=\mathcal{E}^{\otimes m}$ and thus $\tilde{\rho}_{A B}=\rho_{A B}^{\otimes m}$ and $\tilde{\rho}_{A B}=\rho_{A B}^{\otimes m}$ we are able to invoke the estimate

$$
\frac{1}{m} H_{\min }^{\kappa}\left(\rho_{A B}^{\otimes m} \mid \rho^{\otimes m}\right) \geq H\left(\rho_{A B}\right)-H(\rho)-3 \lambda,
$$

where $\lambda(\kappa, m) \rightarrow 0$ as $m \rightarrow \infty$ [34] in order to conclude that $Q$ is not a $\left(n m, a_{m}, b_{m}\right)-\mathrm{QBSC}_{m[\chi(\mathcal{E})+2 \lambda]}$ if $a_{m}+b_{m}+\delta<m n$. This shows that if $P$ is a $\left(n m, a_{m}, b_{m}\right)-\mathrm{QBSC}_{m[\chi(\mathcal{E})+2 \lambda]}$ with $\alpha_{m} m$ $+\beta_{m} m \leq a_{m}+b_{m}<n m-\delta$, i.e., $\alpha_{m}+\beta_{m}<n-\delta / m$, then its $m$-fold execution cannot be secure. Taking $m$ to infinity we see that if $P$ is an $(n, a, b)-\mathrm{QBSC}_{\chi}$ with $a+b<n$ then it cannot be executed securely an arbitrary number of times in parallel.

It follows directly from Ref. [37] that the results in this section also hold in the presence of superselection rules.

\section{POSSIBILITY}

Surprisingly, if one is willing to measure Bob's ability to learn $x$ using the accessible information, nontrivial protocols become possible. These protocols are based on a discovery known as "locking of classical information in quantum states" [18].

\section{Family of protocols}

The protocol, which we call LOCKCOM $(n, \mathcal{U})$, uses this effect and is specified by a set $\mathcal{U}=\left\{U_{1}, \ldots, U_{|\mathcal{U}|}\right\}$ of unitaries.

Commit phase. Alice has the string $x \in\{0,1\}^{n}$ and randomly chooses $r \in\{1, \ldots,|\mathcal{U}|\}$. She sends the state $U_{r}|x\rangle$ to Bob, where $U_{r} \in \mathcal{U}$.

Reveal phase. Alice announces $r$ and $x$. Bob applies $U_{r}^{\dagger}$ and measures in the computational basis to obtain $x^{\prime}$. He accepts if and only if $x^{\prime}=x$.

We first show that our protocol is secure with respect to definition 1 if Alice is dishonest. Note that our proof only depends on the number of unitaries used, and is independent of a concrete instantiation of the protocol.

Lemma 4. Any LOCKCOM $(n, \mathcal{U})$ protocol is $\log _{2}(|\mathcal{U}|)$-binding, i.e., $2^{a} \leq|\mathcal{U}|$.

Proof. Let $p_{x}^{A}$ denote the probability that Alice reveals $x$ successfully. Then, $p_{x}^{A} \leq \Sigma_{r} p_{x, r}^{A}$, where $p_{x, r}^{A}$ is the probability that $x$ is accepted by Bob when the reveal information was $r$. Let $\rho$ denote the state of Bob's system. Summation over $x$ now yields

$$
\sum_{x} p_{x}^{A} \leq \sum_{x, r} p_{x, r}^{A}=\sum_{x, r} \operatorname{Tr}|x\rangle\left\langle x\left|U_{r}^{\dagger} \rho U_{r}=\sum_{r} \operatorname{Tr} \rho=\right| \mathcal{U}\right|,
$$

hence $a \leq \log _{2}|\mathcal{U}|$.

In order to examine security against a dishonest Bob, we have to consider the actual form of the unitaries. We first show that there do indeed exist interesting protocols. Second, we present a simple, implementable, protocol. To see that interesting protocols can exist, let Alice choose a set of $O\left(n^{4}\right)$ unitaries independently according to the Haar measure (approximated) and announce the resulting set $\mathcal{U}$ to Bob. They then perform LOCKCOM $(n, \mathcal{U})$. Following the work of Ref. [38], we now show that this variant is secure against Bob with high probability in the sense that there exist $O\left(n^{4}\right)$ unitaries that bring Bob's accessible information down to a constant: $I_{\text {acc }}(\mathcal{E}) \leq 4$

Theorem 3. For $n \geq 3$, there exist $\left[n, 4 \log _{2} n\right.$ $+O(1), 4]-\mathrm{QBSC}_{I_{\text {acc }}}$ protocols.

Proof. Let $\mathcal{U}_{\text {ran }}$ denote the set of $m$ randomly chosen bases and consider the LOCKCOM $\left(n, \mathcal{U}_{\text {ran }}\right)$ scheme using unitaries $\mathcal{U}=\mathcal{U}_{\text {ran. }}$ Security against Alice is again given by lemma 4. We now need to show that this choice of unitaries achieves the desired locking effect and thus security against Bob. Again, let $d=2^{n}$ denote the dimension. It was observed in Ref. [18] that

$$
I_{\mathrm{acc}} \leq \log _{2} d+\max _{|\phi\rangle} \sum_{i} \frac{1}{m} H\left(X_{j}\right)
$$

where $X_{j}$ denotes the outcome of the measurement of $|\phi\rangle$ in basis $j$ and the maximum is taken over all pure states $|\phi\rangle$. According to Ref. [38] (Appendix B) there is a constant $C^{\prime}$ $>0$ such that 


$$
\begin{aligned}
\operatorname{Pr}\left[\underset{|\phi\rangle}{\inf \frac{1}{m} \sum_{j=1}^{m} H\left(X_{j}\right)}\right. & \left.\leq(1-\epsilon) \log _{2} d-3\right] \\
& \leq\left(\frac{10}{\epsilon}\right)^{2 d} 2^{-m\left[\epsilon C^{\prime} d / 2\left(\log _{2} d\right)^{2}-1\right]}
\end{aligned}
$$

for $d \geq 7$ and $\epsilon \leq 2 / 5$. Set $\epsilon=\frac{1}{\log _{2} d}$. The right-hand side of the above equation then decreases provided that $m$ $>\frac{8}{C^{\prime}}\left(\log _{2} d\right)^{4}$. Thus with $d=2^{n}$ and $\log _{2} m=4 \log _{2} n+O(1)$, the accessible information is then $I_{\text {acc }} \leq \log _{2} d-(1-\epsilon) \log _{2} d$ $+3=\epsilon \log _{2} d+3=4$ for our choice of $\epsilon$.

Unfortunately, the protocol is inefficient both in terms of computation and communication. It remains open to find an efficient constructive scheme with those parameters.

In contrast, for only two bases, an efficient construction exists and uses the identity and the Hadamard transform as unitaries. For this case, the security of the standard LOCKCOM protocol follows immediately:

Theorem 4. LOCKCOM $\left(n,\left\{I^{\otimes n}, H^{\otimes n}\right\}\right)$ is a $(n, 1, n / 2)$ $-\mathrm{QBSC}_{I_{\text {acc }}}$ protocol.

Proof. It is sufficient to apply lemma 4 and the fact that for Bob $I_{\text {acc }} \leq n / 2[18,39]$.

\section{CHEAT-SENSITIVE PROTOCOL}

\section{A. Cheat sensitivity}

We now modify the protocol LOCKCOM $\left(n,\left\{I^{\otimes n}, H^{\otimes n}\right\}\right)$ so that it becomes cheat sensitive against Bob. That is, even though Bob has the possibility to gain information about the committed string before the reveal phase, Alice has a decent probability of catching Bob if he actually obtains a nonzero amount of information $[46,47]$. The modified protocol will be denoted by CS-Bob-LOCKCOM $\left(n,\left\{\mathbb{I}^{\otimes n}, H^{\otimes n}\right\}\right)$.

Commit phase. Alice has the string $x \in\{0,1\}^{n}$ and randomly chooses $r \in\{0,1\}$. She sends the state $|x\rangle$ to Bob if $r=0$ and she sends $H^{\otimes n}|x\rangle$ if $r=1$.

Reveal phase. Alice announces $r$. Bob applies $H^{\otimes n}$ if $r$ $=1$ and does nothing if $r=0$. He then measures in the computational basis to obtain $x^{\prime}$.

Confirmation phase. Bob sends $x^{\prime}$ to Alice. If Alice is honest she declares "accept" if $x=x^{\prime}$ and otherwise "abort."

As before, the cheat parameters are $a=1$ and $b=\frac{n}{2}$. The definition of CS-Bob-LOCKCOM $(n, \mathcal{U})$ is analogous.

Note that a cheating Bob can deviate from the protocol without being caught. This is so because any unitary transformation plus the attachment of an ancilla by Bob is, by definition, reversible. So, the focus has to be on the case where Bob performs an irreversible operation. In quantum mechanics, this corresponds to Bob performing a generalized measurement (POVM).

Now, whenever Bob performs a generalized measurement, the situation can be equivalently formulated by Bob splitting his system $Y$ into $C$ and $Q$ where $C$ is classical and cannot be "touched" by Bob later on. Of course, if $C$ contains no information whatsoever about Alice's commitment, Bob cannot be caught. So, the interesting question to ask is the case when $C$ does contain information about Alice's commitment. In our paper, we quantify the amount of cheating by Bob by the amount of classical mutual information the system $C$ contains about Alice's commitment.

Recall that for any measurement that Bob performs on his quantum state before the reveal phase, his outcome random variable $C$ will obey

$$
I(X ; C) \leq \frac{n}{2}
$$

This bound can be achieved for a measurement in the computational basis. If Bob actually performs this measurement, then he obtains the correct string if $r=0$, i.e., with probability one half, and a random and completely uncorrelated result if $r=1$. It is then easy to see that Alice will abort in the confirmation phase with probability one half, i.e., she is able to detect Bob with rather high probability.

The results in this section will imply that if Bob performs any measurement with outcome random variable $C$ where $I(X ; C)>0$, then Alice will detect him with strictly positive probability (corollary 1). In analogy to results regarding bit commitment $[28,29]$ we will therefore say that CS-BobLOCKCOM $\left(n,\left\{I^{\otimes n}, H^{\otimes n}\right\}\right)$ is cheat sensitive against Bob. In the following we derive an information-gain vs detection tradeoff which implies the mentioned cheat-sensitivity result.

\section{B. Information-gain vs detection}

Before we start explaining the tradeoff, recall that theorem 4 states that CS-Bob-LOCKCOM $\left(n,\left\{I^{\otimes n}, H^{\otimes n}\right\}\right)$ is an $(n, 1, n / 2)-I_{\text {acc }}$-quantum string commitment protocol. This result can be extended to dimensions different from $d=2^{n}$ : one can show (using Ref. [39], corollary 3) that CS-BobLOCKCOM $\left(\log _{2} d,\{\mathbb{I}, U\}\right)$, where $U$ is the Fourier transform, is a $\left(\log _{2} d, 1, \frac{\log _{2} d}{2}\right)-I_{a c c}$-quantum string commitment protocol. The results presented in the following will concern this larger class of protocols.

In Sec. IV we quantified Bob's cheating as the mutual information of the committed string $X$ and the outcome random variable $C$ of a measurement before the reveal phase. We can model this measurement process as a unitary evolution $V_{\text {cheat }}$ that splits the system $Y$ that Bob obtains from Alice during the commit phase into $C$ and $Q$. Since $C$ is classical we can assume without loss of generality that $Q$ contains a copy of $C$. Any further action by Bob during the protocol can thus be assumed not to involve $C$.

In the following it will prove handy to lift the restriction of classicality on system $C$ and only assume that the unitary $V_{\text {cheat }}$ splits $Y$ into $C$ and $Q$ and that Bob will not touch $C$ during the rest of the protocol. Since this only increases Bob's power, any proof of cheat-sensitivity in this scenario will imply cheat sensitivity in the weaker scenario. The mutual information $I(X ; C)$ will correspondingly be generalized to the Holevo information between $X$ and $C: \chi\left(\mathcal{E}^{C}\right)$, where $\mathcal{E}^{C}=\left\{p_{x}, \rho_{x}^{C}\right\}$ denotes Bob's ensemble (with respect to $X$ ) in register $C$. Our main result is the following information-gain vs detection tradeoff.

Theorem 5. If Alice and Bob run the protocol CS-BobLOCKCOM $\left(\log _{2} d,\{I, U\}\right)$ and if Bob is detected cheating with probability less than $p$, then the Holevo information in register $C$ obeys 


$$
\begin{array}{l|l|l|l|}
R & X & R^{\prime} & Y \\
\hline
\end{array}
$$

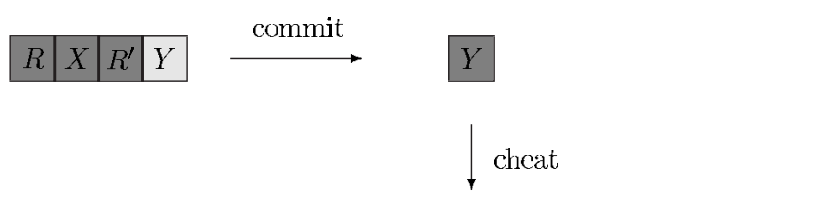

$R|X| R^{\prime} \quad \quad C \mid Q$

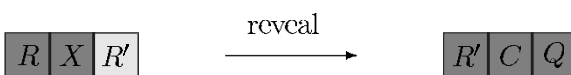
prepare

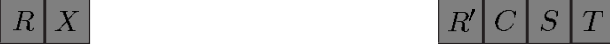

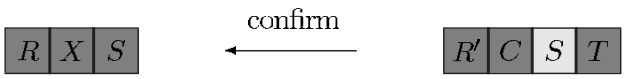

FIG. 2. Execution of CS-Bob-LOCKCOM with honest Alice on the left and cheating Bob on the right. Time flows downwards.

$$
\chi\left(\mathcal{E}^{C}\right) \leq 4 \sqrt{p} \log _{2} d+2 \mu(2 \sqrt{p}) .
$$

As an immediate corollary we find that CS-Bob-LOCKCOM $\left(\log _{2} d,\{\mathbb{I}, U\}\right)$ and, in particular, CS-Bob-LOCKCOM $\left(n,\left\{I^{\otimes n}, H^{\otimes n}\right\}\right)$ is cheat sensitive against Bob.

Corollary 1. If Alice and Bob run the protocol CS-BobLOCKCOM $\left(\log _{2} d,\{\mathbb{I}, U\}\right)$, if Bob performs a measurement before the reveal phase and if his outcome random variable $C$ obeys $I(X ; C)>0$, then he will be detected by Alice with probability $p>0$.

\section{Proof of theorem 5}

We start this section with a description of the sequence of events for the case where Alice is honest and Bob applies a general cheating strategy as outlined above (see also Fig. 2).

The commit phase of the protocol LOCKCOM $\left(\log _{2} d,\{I, U\}\right)$ is equivalent to the following procedure: Alice prepares the state

$$
|\psi\rangle=\frac{1}{\sqrt{2 d}} \sum_{x, r}|x\rangle^{X}|r\rangle^{R}|r\rangle^{R^{\prime}} U^{r}|x\rangle^{Y}
$$

on the system $X R Y R^{\prime}$ and sends system $Y$ (over a noiseless quantum channel) to Bob. It is understood that $U^{0}=I$ and $U^{1}=U$. Note that $R^{\prime}$ contains an identical copy of $R$ and corresponds to the reveal information.

Bob's most general cheating operation can be described by a unitary matrix $V_{\text {cheat }}$ that splits the system $Y$ into $C$ and
Q. $C$ contains by definition the information gathered during cheating and is not touched upon later on

$$
V_{\text {cheat }}: Y \rightarrow C Q \text {. }
$$

The map $V_{\text {cheat }}$ followed by the partial trace over $Q$ is denoted by $\Lambda^{C}$ and likewise $V_{\text {cheat }}$ followed by the partial trace over $C$ is denoted by $\Lambda^{Q}$. Alice sends the reveal information $R^{\prime}$ to Bob. Bob applies a preparation unitary $V_{\text {prepare }}$ to his system. Since $C$ will not be touched upon, the most general operation acts on $R^{\prime} Q$ only:

$$
V_{\text {prepare }}: R^{\prime} Q \rightarrow R^{\prime} S T \text {. }
$$

Bob then sends $S$ to Alice and keeps $T$. Alice measures $S$ in the computational basis and compares the outcome to her value in $X$. If the values do not agree, we say that Alice has detected Bob cheating. The probability for this happening is given by

$$
\frac{1}{d} \sum_{x=1}^{d}\left(1-\operatorname{Tr}|x\rangle\langle x| \rho_{x}^{S}\right)
$$

where $\rho_{x}^{S}=\operatorname{Tr}_{X R R^{\prime} T}|x\rangle\langle x \| \psi\rangle\left\langle\left\langle\left.\psi\right|^{X R R^{\prime} S T} \text { and } \mid \psi\right\rangle^{X R R^{\prime} S T}\right.$ is the pure state of the total system after Bob's application of $V_{\text {prepare }}$. Note that Alice measures in the computational basis since for honest Bob $V_{\text {prepare }}=\Sigma_{r^{\prime} \in\{0,1\}}\left|r^{\prime}\right\rangle\left\langle r^{\prime}\right| \otimes\left(U^{r}\right)^{\dagger}$, in which case his outcome agrees with the committed value of an honest Alice.

Before we start with the proof of theorem 5, we define ensembles depending on the classical information contained in $X R$, i.e., for $Z \in\{C, Q\}$, define $\mathcal{E}_{r}^{Z}=\left\{p_{x}, \rho_{x r}^{Z}\right\}$ with

$$
\rho_{x r}^{Z}=\frac{1}{p_{x} p_{r}} \operatorname{Tr}_{X R R^{\prime} C Q \backslash Z}|x r\rangle\langle x r \| \psi\rangle\left\langle\left\langle\left.\psi\right|^{X R R^{\prime} C Q}\right.\right.
$$

and for $Z \in\{S, T\}$ let $\mathcal{E}_{r}^{Z}=\left\{p_{x}, \rho_{x r}^{Z}\right\}$ with

$$
\rho_{x r}^{Z}=\operatorname{Tr}_{X R R^{\prime} C S T Z Z}|x r\rangle\langle x r \| \psi\rangle\left\langle\psi||^{X R R^{\prime} C S T} .\right.
$$

Sometimes we are only interested in the ensemble averaged over the values of $r$ : for $Z \in\{C, Q, S, T\}$

$$
\mathcal{E}^{Z}=\left\{p_{x}, \rho_{x}^{Z}\right\}, \text { where } \rho_{x}^{Z}=\frac{1}{2}\left(\rho_{x 0}^{Z}+\rho_{x 1}^{Z}\right) .
$$

We now come to two technical lemmas, most notably a channel uncertainty relation (lemma 5) that was discovered in connection with squashed entanglement: Consider a uniform ensemble $\mathcal{E}_{0}=\left\{\frac{1}{d},|i\rangle\right\}_{i=1}^{d}$ of basis states of a Hilbert space $\mathcal{H}$ and the ensemble $\mathcal{E}_{1}=\left\{\frac{1}{d}, U|i\rangle\right\}_{i=1}^{d}$ rotated with a unitary $U$. Application of the completely positive trace preserving (CPTP) map $\Lambda$ (with output in a potentially different Hilbert space) results in the two ensembles

$$
\Lambda\left(\mathcal{E}_{0}\right)=\left\{\frac{1}{d}, \Lambda(|i\rangle\langle i|)\right\}, \quad \Lambda\left(\mathcal{E}_{1}\right)=\left\{\frac{1}{d}, \Lambda\left(U|i\rangle\langle i| U^{\dagger}\right)\right\}
$$

with Holevo information for $\mathcal{E}_{0}$ given by 


$$
\chi\left(\Lambda\left(\mathcal{E}_{0}\right)\right)=H\left(\frac{1}{d} \sum_{i} \Lambda(|i\rangle\langle i|)\right)-\frac{1}{d} \sum_{i} H[\Lambda(|i\rangle\langle i|)]
$$

and similarly for $\mathcal{E}_{1}$. Consider also the quantum mutual information of $\Lambda$ relative to the maximally mixed state $\tau=\frac{1}{d} \mathbb{I}$, which is the average state of either $\mathcal{E}_{0}$ or $\mathcal{E}_{1}$ :

$$
I(\tau ; \Lambda)=H(\tau)+H[\Lambda(\tau)]-H\left[(\mathbb{I} \otimes \Lambda)\left(\left|\psi_{d}\right\rangle\left\langle\psi_{d}\right|\right)\right],
$$

where $\left|\psi_{d}\right\rangle$ is a maximally entangled state in dimension $d$ purifying $\tau$.

Lemma 5 (Channel uncertainty relation [[39]]). Let $U$ be the Fourier transform of dimension $d$, i.e., of the Abelian group $Z_{d}$ of integers modulo $d$. More generally, $U$ can be a Fourier transform of any finite Abelian group labeling the ensemble $\mathcal{E}_{0}$, e.g., for $d=2^{l}$, and the group $\mathbb{Z}_{2}^{l}, U=H^{\otimes l}$ with the Hadamard transform $H$ of a qubit. Then for all CPTP maps $\Lambda$,

$$
\chi\left(\Lambda\left(\mathcal{E}_{0}\right)\right)+\chi\left(\Lambda\left(\mathcal{E}_{1}\right)\right) \leq I(\tau ; \Lambda) .
$$

The following technical lemma is a technical consequence of Fannes' inequality.

Lemma 6. Let $\mathcal{E}=\left\{p_{i}, \rho_{i}=\left|\psi_{i}\right\rangle\left\langle\psi_{i}\right|\right\}$ be an ensemble of pure states and $\widetilde{\mathcal{E}}=\left\{p_{i}, \sigma_{i}\right\}$ be an ensemble of mixed states, both on $\mathrm{C}^{d}$. If $\sum_{i} p_{i}\left\langle\psi_{i}\left|\sigma_{i}\right| \psi_{i}\right\rangle \geq 1-p$, then

$$
|\chi(\widetilde{\mathcal{E}})-\chi(\mathcal{E})| \leq 4 \sqrt{p} \log _{2} d+2 \mu(2 \sqrt{p}),
$$

where $\mu(x)=\min \left\{-x \log _{2} x, \frac{1}{e}\right\}$.

Proof. The justification of the estimate

$$
p \geq \sum_{i} p_{i}\left(1-\operatorname{Tr} \rho_{i} \sigma_{i}\right) \geq \sum_{i} p_{i} \delta_{i}^{2} \geq\left(\sum_{i} p_{i} \delta_{i}\right)^{2},
$$

where $\delta_{i}=\delta\left(\rho_{i}, \sigma_{i}\right)$ is as follows: the second inequality is a standard relation between the fidelity and the trace distance and the third follows from the convexity of the square function. Strong convexity of the trace distance implies $\delta(\rho, \sigma)$ $\leq \sqrt{p}$. Fannes' inequality will be applied to the overall state

$$
|H(\rho)-H(\sigma)| \leq 2 \sqrt{p} \log _{2} d+\min \left\{\eta(2 \sqrt{p}), \frac{1}{e}\right\},
$$

where $\eta(x)=-x \log _{2} x$, and to the individual ones

$$
\begin{aligned}
\sum_{i} p_{i}\left|H\left(\sigma_{i}\right)-H\left(\rho_{i}\right)\right| \leq & \left(\sum_{i} p_{i} \delta_{i}\right) 2 \log _{2} d \\
& +\sum_{i} p_{i} \min \left\{\eta\left(2 \delta_{i}\right), \frac{1}{e}\right\} \\
\leq & \sqrt{p} 2 \log _{2} d+\min \left\{\eta(2 \sqrt{p}), \frac{1}{e}\right\},
\end{aligned}
$$

where the last inequality is true by the concavity of $\eta(x)$. Inserting these estimates in the Holevo $\chi$ quantities $\chi(\mathcal{E})$ $=H(\rho)$ and $\chi(\widetilde{\mathcal{E}})=H(\sigma)-\Sigma_{i} p_{i} H\left(\sigma_{i}\right)$ concludes the proof.

Proof [Proof of theorem 5]. Let $\mathcal{E}_{0}$ and $\mathcal{E}_{1}$ be defined as in lemma 5. In the commit phase of the protocol, Alice chooses one of the ensembles (each with probability $\frac{1}{2}$ ), and one of the states in the ensemble (each with probability $\frac{1}{d}$ ). The justifications for the following estimate are given in a list below:

$$
\begin{aligned}
\chi\left(\mathcal{E}_{0}^{C}\right) & +\chi\left(\mathcal{E}_{1}^{C}\right), \\
& =\chi\left(\Lambda^{C}\left(\mathcal{E}_{0}\right)\right)+\chi\left(\Lambda^{C}\left(\mathcal{E}_{1}\right)\right), \\
& \leq I\left(X R R^{\prime} ; C\right), \\
& =2 H\left(X R R^{\prime}\right)-I\left(X R R^{\prime} ; Q\right), \\
& \leq 2 H\left(X R R^{\prime}\right)-\chi\left(\Lambda^{Q}\left(\mathcal{E}_{0}\right)\right)-\chi\left(\Lambda^{Q}\left(\mathcal{E}_{1}\right)\right), \\
& =2 H(X R)-\chi\left(\mathcal{E}_{0}^{Q}\right)-\chi\left(\mathcal{E}_{1}^{Q}\right), \\
& \leq 2 H(X R)-\chi\left(\Lambda_{0}^{S}\left(\mathcal{E}_{0}^{Q}\right)\right)-\chi\left(\Lambda_{1}^{S}\left(\mathcal{E}_{1}^{Q}\right)\right), \\
& =2 H(X R)-\chi\left(\mathcal{E}_{0}^{S}\right)-\chi\left(\mathcal{E}_{1}^{S}\right), \\
& \leq 2 H(X R)-2 \chi\left(\mathcal{E}^{S}\right) .
\end{aligned}
$$

The justifications. Equality (8): By definition of the string commitment scheme and the map $\Lambda^{C}: \mathcal{E}_{r}^{C}=\left\{p_{x}, \rho_{x r}^{C}\right\}$ $=\left\{p_{x}, \Lambda^{C}\left[U^{r}|x\rangle\langle x|\left(U^{\dagger}\right)^{r}\right]\right\}=: \Lambda^{C}\left(\mathcal{E}_{r}\right)$. Inequality (9): Application of lemma 5 for the map $\Lambda^{C}$. Note that system $X R R^{\prime}$ is a reference system for the completely mixed state on system $Y$ on which the channel $\Lambda^{C}$ is applied. Hence $I\left(\tau ; \Lambda^{C}\right)$ $=I\left(X R R^{\prime} ; C\right)$. Equality $(10)$ : Simple rewriting of the entropy terms making use of the definition of quantum mutual information and the purity of $X R R^{\prime} C Q$. Inequality (11): Application of lemma 5 for the map $\Lambda^{Q}$. Note that system $X R R^{\prime}$ is a reference system for the completely mixed state on system $Y$ on which the channel $\Lambda^{Q}$ is applied. Hence $I\left(\tau ; \Lambda^{Q}\right)$ $=I\left(X R R^{\prime} ; Q\right)$. Equality $(12): R^{\prime}$ is a copy of $R: H\left(X R R^{\prime}\right)$ $=H(X R)$. By definition of the string commitment scheme and the map $\Lambda^{Q}: \mathcal{E}_{r}^{Q}=\left\{p_{x}, \rho_{x r}^{Q}\right\}=\left\{p_{x}, \Lambda^{Q}\left[U^{r}|x\rangle\langle x|\left(U^{\dagger}\right)^{r}\right]\right\}$. Inequality (13) and equality (14): follow from the data processing inequality $\chi\left(\Lambda^{H}\left(\mathcal{E}_{r}^{Q}\right)\right) \leq \chi\left(\mathcal{E}_{r}^{Q}\right)$ and from the definition $\Lambda^{H}\left(\mathcal{E}_{r}^{Q}\right)=\mathcal{E}_{r}^{S}$. Inequality (15): Finally $\mathcal{E}^{S}=\left\{p_{x}, \rho_{x}^{S}=\frac{1}{2}\left(\rho_{x 0}^{S}\right.\right.$ $\left.\left.+\rho_{x 1}^{S}\right)\right\}$, which by the concavity of von Neumann entropy implies $\chi\left(\mathcal{E}^{S}\right) \leq \frac{1}{2}\left[\chi\left(\mathcal{E}_{0}^{S}\right)+\chi\left(\mathcal{E}_{1}^{S}\right)\right]$.

If Bob is detected cheating with probability less than $p$, then by lemma 6 the Holevo quantity $\chi\left(\mathcal{E}^{S}\right)$ of the ensemble given in $S$ that Bob sends to Alice obeys

$$
\chi\left(\mathcal{E}^{S}\right) \geq(1-4 \sqrt{p}) \log _{2} d-2 \mu(2 \sqrt{p}) .
$$

Inserting inequality (16) into inequality (15) and noting that $H(X R)=H(Y)=\log _{2} d$ proves the claim.

This proves cheat-sensitivity against Bob for the simplest protocol of the LOCKCOM family.

\section{CONCLUSION}

We have introduced a framework for quantum commitments to a string of bits. Even though string commitments are weaker than bit commitments, we showed that under strong security requirements, there are no such nontrivial 
protocols. A property of quantum states known as locking, however, allowed us to propose meaningful protocols for a weaker security demand. Since the completion of our original work [40], Tsurumaru [41] has also proposed a different QBSC protocol within our framework.

Furthermore, we have shown that one such protocol can be made cheat sensitive. It is an interesting open question to derive a tradeoff between Bob's ability to gain information and Alice's ability to detect him cheating for the protocol of theorem 3 as well.

A drawback of weakening the security requirement is that LOCKCOM protocols are not necessarily composable. Thus, if LOCKCOM is used as a subprotocol in a larger protocol, the security of the resulting scheme has to be evaluated on a case by case basis. However, LOCKCOM protocols are secure when executed in parallel. This is a consequence of the definition of Alice's security parameter and the additivity of the accessible information [42,43], and sufficient for many cryptographic purposes.

Nonetheless, two important open questions remain. First, how can we construct efficient protocols using more than two bases? It may be tempting to conclude that we could simply use a larger number of mutually unbiased bases, such as given by the identity and Hadamard transform. Yet, it has been shown [44] that using more mutually unbiased bases does not necessarily lead to a better locking effect and thus better string commitment protocols. Second, are there any real-life applications for this weak quantum string commitment?

\section{ACKNOWLEDGMENTS}

We thank J. Barrett, A. Broadbent, I. Damgård, A. Kent, S. Massar, R. Renner, R. Spekkens, and R. de Wolf for discussions. We also thank R. Jain for discussion on his work [45], where, following our preprint [40], he used a different method to prove that $(n, a, b)-\mathrm{QBSC}_{\chi} \mathrm{s}$ with $a+16 b+31<n$ do not exist. We thank the German Academic Exchange Service, the U.K. EPSRC, the Magdalene College Cambridge, CFI, CIFAR, CIPI, CRC, NSERC, PREA, and OIT, the NWO vici Project No. 2004-2009, EU Project No. RESQ IST-2001-37559, QAP IST Grant No. 015848, the FP6-FET Integrated Project SCALA, Grant No. CT-015714, the Sloan Foundation, QuantumWorks, and NSF Grant No. PHY0456720 .
[1] C. H. Bennett, G. Brassard, C. Crépeau, and M.-H. Skubiszewska, in CRYPTO '91: Proceedings of the 11th Annual International Cryptology Conference on Advances in Cryptology (Springer-Verlag, Berlin, 1992), pp. 351-366.

[2] A. C.-C. Yao, in Proceedings of 20th ACM STOC (ACM, New York, 1995), pp. 67-75.

[3] C. Crépeau, J. Mod. Opt. 41, 2445 (1994).

[4] C. Crépeau, J. van de Graaf, and A. Tapp, in CRYPTO '95: Proceedings of the 15th Annual International Cryptology Conference on Advances in Cryptology (Springer-Verlag, Berlin, 1995), pp. 110-123.

[5] J. Kilian, in Proceedings of 20th ACM STOC (ACM, New York, 1988), pp. 20-31.

[6] O. Goldreich, Foundations of Cryptography (Cambridge University Press, Cambridge, 2001), Vol. Basic Tools.

[7] M. Blum, SIGACT News 15, 23 (1983).

[8] C. H. Bennett and G. Brassard, in Proceedings of the IEEE International Conference on Computers, Systems and Signal Processing (IEEE, New York, 1984), pp. 175-179.

[9] G. Brassard and C. Crépeau, in Advances in CryptologyProceedings of Crypto '90 (Springer-Verlag, New York, 1990), pp. 49-61.

[10] G. Brassard, C. Crépeau, R. Jozsa, and D. Langlois, in Proceedings of 34th IEEE FOCS (IEEE, New York, 1993), pp. 362-371.

[11] D. Mayers, e-print arXiv:quant-ph/9603015.

[12] H.-K. Lo and H. F. Chau, Phys. Rev. Lett. 78, 3410 (1997).

[13] D. Mayers, Phys. Rev. Lett. 78, 3414 (1997).

[14] H.-K. Lo and H. Chau, Physica D 120, 177 (1998).

[15] G. Brassard, C. Crépeau, D. Mayers, and L. Salvail, e-print arXiv:quant-ph/9712023.

[16] H. Chau and H.-K. Lo, Fortschr. Phys. 46, 507 (1998).

[17] R. W. Spekkens and T. Rudolph, Phys. Rev. A 65, 012310
(2001).

[18] D. DiVincenzo, M. Horodecki, D. Leung, J. Smolin, and B. Terhal, Phys. Rev. Lett. 92, 067902 (2004).

[19] L. Salvail, in Proceedings of CRYPTO'98 Lecture Notes in Computer Science Vol. 1462, p. 338 (1998).

[20] D. DiVincenzo, J. Smolin, and B. Terhal, New J. Phys. 6, 80 (2004).

[21] I. Damgård, S. Fehr, L. Salvail, and C. Schaffner, in Proceedings of 46th IEEE FOCS (IEEE, New York, 2005), pp. 449458.

[22] I. Damgard, S. Fehr, R. Renner, L. Salvail, and C. Schaffner, in Proceedings of CRYPTO 2007 (Springer-Verlag, New York, 2007), pp. 360-378.

[23] S. Wehner and J. Wullschleger, e-print arXiv:0709.0492.

[24] S. Wehner, C. Schaffner, and B. Terhal, Phys. Rev. Lett. 100, 220502 (2008).

[25] C. Cachin, C. Crépeau, and J. Marcil, in Proceedings of 39th IEEE FOCS (IEEE, New York, 1998), pp. 493-502.

[26] C. Crépeau and J. Kilian, in Proceedings of 29th IEEE FOCS (IEEE, New York, 1988), pp. 42-52.

[27] A. Winter, A. Nascimento, and H. Imai, in Proceedings of 9th Cirencester Crypto and Coding, Vol. 2989 of Lecture Notes in Computer Science (Springer, Berlin, 2003).

[28] L. Hardy and A. Kent, Phys. Rev. Lett. 92, 157901 (2004).

[29] D. Aharonov, A. Ta-Shma, U. Vazirani, and A. Yao, in Proceedings of the 32th ACM STOC (ACM, New York, 2000), pp. $705-714$.

[30] A. Kent, J. Cryptology 18, 313 (2005).

[31] A. Kent, Phys. Rev. Lett. 90, 237901 (2003).

[32] A. Kent, (private communication).

[33] T. Tsurumaru, Phys. Rev. A 71, 012313 (2005).

[34] R. Renner, Ph.D. thesis, ETH Zurich, Zurich, 2005, e-print arXiv:quant-ph/0512258. 
[35] H. Barnum and E. Knill, J. Math. Phys. 43, 2097 (2002).

[36] R. König, U. Maurer, and R. Renner, IEEE Trans. Inf. Theory 51, 2391 (2005).

[37] A. Kitaev, D. Mayers, and J. Preskill, Phys. Rev. A 69, 052326 (2004).

[38] P. Hayden, D. Leung, P. Shor, and A. Winter, Commun. Math. Phys. 250, 371 (2004).

[39] M. Christandl and A. Winter, IEEE Trans. Inf. Theory 51, 3159 (2005).

[40] H. Buhrman, M. Christandl, P. Hayden, H.-K. Lo, and S. Wehner, e-print arXiv:quant-ph/0504078.
[41] T. Tsurumaru, Phys. Rev. A 74, 042307 (2006).

[42] A. S. Holevo, Probl. Inf. Transm. 9, 110 (1973).

[43] D. P. DiVincenzo, D. W. Leung, and B. M. Terhal, IEEE Trans. Inf. Theory 48, 580 (2002).

[44] M. A. Ballester and S. Wehner, Phys. Rev. A 75, 022319 (2007).

[45] R. Jain, e-print arXiv:quant-ph/0506001.

[46] M. Christandl, Ph.D. thesis, University of Cambridge, Cambridge, 2005, e-print arXiv:quant-ph/0604183.

[47] The results in this section are included in Ref. [46]. 